\title{
Effect of Dates of Transplanting on Incidence of Sucking Pests in Chilli, Capsicum annum L.
}

\author{
Amit Kumar Yadav ${ }^{1^{*}}$ and V. S. Acharya²
}

${ }^{1}$ Rajasthan Agricultural Research Institute Durgapura, Jaipur, Rajasthan (302 018), India

${ }^{2}$ College of Agriculture, Bikaner, Rajasthan (334 006), India

\section{Corresponding Author}

Amit Kumar Yadav

e-mail: amitvidrohi002@gmail.com

\author{
Article History \\ Article ID: 3 C1060 \\ Received in $06^{\text {th }}$ November, 2017 \\ Received in revised form $19^{\text {th }}$ February, 2018 \\ Accepted in final form 26 $6^{\text {th }}$ March, 2018
}

\begin{abstract}
The experiment to examine the effect of five dates of transplanting on the incidence of major sucking pests was laid out in a randomized block design with four replications. The sowing of chilli variety $\mathrm{RCH}-1$ was done at 10 days intervals in different nursery beds in landscape nursery. One month old seedlings were transplanted in the plots measuring $3.6 \times 4.5 \mathrm{~m}^{2}$ at ten days interval starting from $15^{\text {th }}$ Feb. to $27^{\text {th }}$ March, 2010. The effect of five dates of transplanting, $15^{\text {th }}$ Feb., $25^{\text {th }}$ Feb., $7^{\text {th }}$ March, $17^{\text {th }}$ March and $27^{\text {th }}$ March were evaluated on occurrence of major sucking pests of chilli i.e. thrips (Scirtothrips dorsalis Hood), whitefly (Bemisia tabaci Genn.), aphid (Aphis gossypii Glover), jassid (Amrasca biguttula biguttula Ishida) and mite (Polyphagotarsonemus latus Banks). The experiment on five dates of transplanting revealed that early transplanting $15^{\text {th }}$ Feb. had the minimum and $27^{\text {th }}$ March had maximum infestation of sucking pests viz., thrips (18.45 per three leaves and 20.30 per three leaves, respectively), whitefly (21.63 per three leaves and 29.50 per three leaves, respectively), aphid (0.43 per three leaves and 14.55 per three leaves, respectively), jassid ( 9.50 per three leaves and 18.63 per three leaves, respectively) and mite ( 2.50 per three leaves and 7.75 per three leaves, respectively). The maximum green chilli yield ( $24.04 \mathrm{q} \mathrm{ha}^{-1}$ ) was obtained from the crop transplanted in $15^{\text {th }}$ Feb. followed by $25^{\text {th }}$ Feb. $\left(21.49 \mathrm{q} \mathrm{ha}^{-1}\right) 7^{\text {th }}$ March $\left(19.09 \mathrm{q} \mathrm{ha} \mathrm{a}^{-1}\right)$. However all these treatments were statistically at par. The minimum yield (14.19 $\left.\mathrm{q} \mathrm{ha}^{-1}\right)$ was recorded in the crop transplanted on $27^{\text {th }}$ March followed by the crop sown on $\left.17^{\text {th }} \mathrm{March}^{(16.75 \mathrm{q} \text { ha }}{ }^{-1}\right)$.
\end{abstract}

Keywords: Dates of transplanting, thrips, whitefly, jassids, aphid, mite, yield

\section{Introduction}

Chilli (Capsicum annuum L.) is the most extensively cultivated spices as well as vegetable crop of the world. There are two main species of chilli viz., Capsicum annum and Capsicum frutescence of which Indian chilli, $C$. annum is an important vegetable crop due to its adaptability to varied climatic conditions. In India the area of chilli was about 7.89 lakh ha with a production of about 13.89 lakh tones (Anonymous 2015). In Rajasthan it occupies about 9778 ha area with an annual production of 12920 tonnes and average productivity of $1321 \mathrm{~kg} \mathrm{ha}^{-1}$ (Anonymous, 2015). But many factors are responsible for low productivity and production with time but the magnitude of insect pest have been reported to damage the chilli crop from sowing to maturity is most important. About 51 insect and 2 mites species, belonging to 27 families and 9 orders were found infesting chilli (Reddy and Puttaswami, 1985). Among these, thrips (Scirtothrips dorsalis Hood), whitefly (Bemisia tabaci Genn.), aphid (Aphis gossypii Glover), jassid (Amrasca biguttula biguttula) and mite (Polyphagotarsonemus latus Banks) are major sucking pests contributing 60 to 75 per cent yield loss in green chilli (Patel and Gupta, 1998). However, the control of these sucking pests was mainly achieved through the use of insecticide but excessive and indiscriminate use of insecticide has created many pests problems like development of resistance to most of available insecticides, a insecticide induced resurgence and disruption of population of predator and parasitoids. This situation warrants search for more effective alternate strategy to manage these pests and overcome the crises.

In integrated pest management a system oriented approach in needed in crop protection practices. In this context a clear understanding of impact of ecological factor on the population dynamics of the concerned cohort is a pre requisite before formulation an I.P.M. schedule. It is needless to cite the importance of the cohort taken in this pursuit i.e. major sucking pests in chilli agro ecosystem. Thus the study on seasonal incidence of major sucking pests is needed to be worked out in relation to environmental conditions prevailing at Bikaner in agro climate zone I C.

\section{Materials and Methods}

The present investigations were conducted at Agronomy farm, 
College of Agriculture, Bikaner during summer 2010. Seeds of chilli variety $\mathrm{RCH}-1$ were sown in the well prepared nursery beds in the landscape nursery for obtaining the seedlings for different experiments. One month old healthy seedlings were taken and transplanted in well prepared experimental plots at row to row spacing of $60 \mathrm{~cm}$ and plant to plant spacing of $45 \mathrm{~cm}$. The experiment to examine the effect of five dates of transplanting $\left(15^{\text {th }}\right.$ Feb, $25^{\text {th }}$ Feb, $7^{\text {th }}$ March, $17^{\text {th }}$ March and $27^{\text {th }}$ March) on the incidence of major sucking pests was laid out in a randomized block design with four replications. The sowing of chilli variety $\mathrm{RCH}-1$ was done at 10 days intervals in different nursery beds in landscape nursery. One month old seedlings were transplanted in the plots measuring $3.6 \times 4.5$ $\mathrm{m}^{2}$ at ten days interval starting from $15^{\text {th }}$ Feb. to $27^{\text {th }}$ March, 2010. The row to row and plant to plant distance of $0.60 \mathrm{~m}$ and $0.45 \mathrm{~m}$ was maintained. The crop was allowed to have natural insect infestation. The observation on population of major sucking pest of chilli were recorded on five randomly selected tagged plants from each experimental plot soon after the appearance of sucking pests and then at weekly interval till harvesting the crop. Green chilli yield was also recorded after harvesting the crop. The data obtained on population of sucking pests from experimental plots were transformed into $v x+0.5$ values and subjected to analysis of variance. The yield $\mathrm{kg}^{-1}$ plot $^{-1}$ was converted into $\mathrm{q} \mathrm{ha} \mathrm{h}^{-1}$ and then statistically analyzed compared by critical difference.

\section{Results and Discussion}

\subsection{Thrips}

The data on the effect of dates of transplanting on incidence of thrips revealed that the population of thrips remained very low up to June in all dates of transplanting (15 $5^{\text {th }}$ Feb, $25^{\text {th }}$ Feb, $7^{\text {th }}$ March, $17^{\text {th }}$ March, $27^{\text {th }}$ March), thereafter, increased gradually and reached to its peak in the month of May and then declined gradually. At the peak, the maximum population of thrips was recorded on the crop transplanted on $27^{\text {th }}$ March and minimum on $15^{\text {th }}$ Feb transplanted crop. On the basis of overall mean, the minimum population of thrips (7.05/three leaves) was observed on the crop transplanted on $15^{\text {th }}$ Feb. followed by $8.26,9.68$ and 10.72 per three leaves on $25^{\text {th }}$ Feb, $7^{\text {th }}$ March and $17^{\text {th }}$ March transplanted crop, respectively (Table 1$)$. The present results are in conformity to these of Hosmani (1982) and Borah and Langthasa (1995) who reported that increase in population of thrips with the increase of planting dates.

\subsection{Whitefly}

The data on the effect of dates of transplanting on incidence of whitefly revealed that population remained very low up to the last week of July in all dates of transplanting. (15 ${ }^{\text {th }}$ Feb, $25^{\text {th }}$ Feb, $7^{\text {th }}$ March, $27^{\text {th }}$ March) thereafter, increased gradually and reached to its peak in the month of May and then declined gradually. At the peak, the maximum population of whitefly was recorded on the crop transplanted on $27^{\text {th }}$ March and minimum $15^{\text {th }}$ Feb. transplanted crop. On the basis of overall mean the minimum population of whitefly (7.91/three leaves) was observed on the crop sown in $15^{\text {th }}$ Feb. followed by 8.93 , 10.32 and $11.62 /$ three leaves on $25^{\text {th }}$ Feb, $7^{\text {th }}$ March and $17^{\text {th }}$ March transplanting crop, respectively and maximum 13.09/ three leaves on $27^{\text {th }}$ March (Table 2). The present findings are in agreement with those of Bishnoi et al. (1996) and Nagargoje et al. (2002) who reported that early sown crop, was not affected by slight development of population of whitefly but as sowing time delayed, the plant become more sensitive to infestation. These present results are not in conformity with those of Giri et al. (1993); Dhawan et al. (1998) who reported that sowing dates did not influence population buildup of whitefly.

\subsection{Jassid}

The population remained very low up to the last week of May

Table 1: Effect of dates of transplanting on the incidence of thrips during summer 2010

\begin{tabular}{|c|c|c|c|c|c|c|c|c|c|c|c|c|c|c|}
\hline \multirow{2}{*}{$\begin{array}{l}\text { Sl. } \\
\text { No. }\end{array}$} & \multirow{2}{*}{$\begin{array}{l}\text { Treat- } \\
\text { ments }\end{array}$} & \multicolumn{12}{|c|}{ Population*/3 leaves in different standard weeks } & \multirow[b]{2}{*}{25} \\
\hline & & 13 & 14 & 15 & 16 & 17 & 18 & 19 & 20 & 21 & 22 & 23 & 24 & \\
\hline 1. & $15^{\text {th }}$ Feb & $\begin{array}{c}6.38 \\
(2.62)^{* *}\end{array}$ & $\begin{array}{c}9.25 \\
(3.12)\end{array}$ & $\begin{array}{c}9.70 \\
(3.19)\end{array}$ & $\begin{array}{l}10.00 \\
(3.24)\end{array}$ & $\begin{array}{l}11.98 \\
(3.53)\end{array}$ & $\begin{array}{l}13.63 \\
(3.76)\end{array}$ & $\begin{array}{l}18.45 \\
(4.35)\end{array}$ & $\begin{array}{l}15.13 \\
(3.95)\end{array}$ & $\begin{array}{c}9.23 \\
(3.12)\end{array}$ & $\begin{array}{c}5.31 \\
(2.41)\end{array}$ & $\begin{array}{c}4.25 \\
(2.18)\end{array}$ & $\begin{array}{c}3.44 \\
(1.98)\end{array}$ & $\begin{array}{c}3.38 \\
(1.97)\end{array}$ \\
\hline 2. & $25^{\text {th }}$ Feb & $\begin{array}{c}7.98 \\
(2.91)\end{array}$ & $\begin{array}{l}11.75 \\
(3.50)\end{array}$ & $\begin{array}{l}12.50 \\
(3.61)\end{array}$ & $\begin{array}{l}13.13 \\
(3.69)\end{array}$ & $\begin{array}{l}13.43 \\
(3.73)\end{array}$ & $\begin{array}{l}15.18 \\
(3.96)\end{array}$ & $\begin{array}{l}20.30 \\
(4.56)\end{array}$ & $\begin{array}{l}16.25 \\
(4.09)\end{array}$ & $\begin{array}{l}10.63 \\
(3.34)\end{array}$ & $\begin{array}{c}6.69 \\
(2.68)\end{array}$ & $\begin{array}{c}3.75 \\
(2.06)\end{array}$ & $\begin{array}{c}4.08 \\
(2.14)\end{array}$ & $\begin{array}{c}3.68 \\
(2.04)\end{array}$ \\
\hline 3. & $7^{\text {th }}$ Mar & $\begin{array}{l}10.10 \\
(3.26)\end{array}$ & $\begin{array}{l}12.63 \\
(3.62)\end{array}$ & $\begin{array}{l}13.81 \\
(3.78)\end{array}$ & $\begin{array}{l}14.50 \\
(3.87)\end{array}$ & $\begin{array}{l}17.50 \\
(4.24)\end{array}$ & $\begin{array}{l}16.88 \\
(4.17)\end{array}$ & $\begin{array}{l}22.93 \\
(4.84)\end{array}$ & $\begin{array}{l}17.00 \\
(4.18)\end{array}$ & $\begin{array}{l}12.56 \\
(3.61)\end{array}$ & $\begin{array}{c}7.44 \\
(2.82)\end{array}$ & $\begin{array}{c}4.38 \\
(2.21)\end{array}$ & $\begin{array}{c}5.49 \\
(2.45)\end{array}$ & $\begin{array}{c}5.45 \\
(2.44)\end{array}$ \\
\hline 4. & $17^{\text {th }} \mathrm{Mar}$ & $\begin{array}{l}11.88 \\
(3.52)\end{array}$ & $\begin{array}{l}13.30 \\
(3.71)\end{array}$ & $\begin{array}{l}12.75 \\
(3.64)\end{array}$ & $\begin{array}{l}14.81 \\
(3.91)\end{array}$ & $\begin{array}{l}20.30 \\
(4.56)\end{array}$ & $\begin{array}{l}19.80 \\
(4.50)\end{array}$ & $\begin{array}{l}24.98 \\
(5.05)\end{array}$ & $\begin{array}{l}18.29 \\
(4.33)\end{array}$ & $\begin{array}{l}14.25 \\
(3.84)\end{array}$ & $\begin{array}{c}8.43 \\
(2.99)\end{array}$ & $\begin{array}{c}4.94 \\
(2.33)\end{array}$ & $\begin{array}{c}7.85 \\
(2.89)\end{array}$ & $\begin{array}{c}5.93 \\
(2.53)\end{array}$ \\
\hline 5. & $27^{\text {th }} \mathrm{Mar}$ & $\begin{array}{l}10.50 \\
(3.32)\end{array}$ & $\begin{array}{l}14.19 \\
(3.83)\end{array}$ & $\begin{array}{l}15.25 \\
(3.97)\end{array}$ & $\begin{array}{l}15.43 \\
(3.99)\end{array}$ & $\begin{array}{l}23.18 \\
(4.87)\end{array}$ & $\begin{array}{l}23.68 \\
(4.92)\end{array}$ & $\begin{array}{l}27.25 \\
(5.27)\end{array}$ & $\begin{array}{l}20.43 \\
(4.57)\end{array}$ & $\begin{array}{l}16.23 \\
(4.09)\end{array}$ & $\begin{array}{l}10.18 \\
(3.27)\end{array}$ & $\begin{array}{c}6.25 \\
(2.60)\end{array}$ & $\begin{array}{c}9.93 \\
(3.23)\end{array}$ & $\begin{array}{c}6.25 \\
(2.60)\end{array}$ \\
\hline \multicolumn{2}{|c|}{ SEm \pm} & 0.07 & 0.07 & 0.11 & 0.09 & 0.05 & 0.04 & 0.07 & 0.04 & 0.06 & 0.05 & 0.08 & 0.05 & 0.05 \\
\hline \multicolumn{2}{|c|}{$\mathrm{CD}(p=0.05)$} & 0.21 & 0.22 & 0.34 & 0.27 & 0.16 & 0.14 & 0.24 & 0.13 & 0.19 & 0.16 & 0.24 & 0.15 & 0.16 \\
\hline
\end{tabular}




\begin{tabular}{|c|c|c|c|c|c|c|c|}
\hline \multirow{2}{*}{$\begin{array}{l}\text { SI. } \\
\text { No. }\end{array}$} & \multirow[t]{2}{*}{ Treatments } & \multicolumn{5}{|c|}{ Population*/3 leaves in different standard weeks } & \multirow{2}{*}{$\begin{array}{c}\text { Over all } \\
\text { mean }\end{array}$} \\
\hline & & 26 & 27 & 28 & 29 & 30 & \\
\hline 1. & $15^{\text {th }}$ Feb & $2.50(1.73)$ & $2.13(1.62)$ & $1.05(1.24)$ & $0.63(1.06)$ & $0.43(0.96)$ & 7.05 \\
\hline 2. & $25^{\text {th }} \mathrm{Feb}$ & $3.25(1.94)$ & $2.75(1.80)$ & $1.38(1.37)$ & $1.05(1.24)$ & $0.88(1.17)$ & 8.26 \\
\hline 3. & $7^{\text {th }}$ Mar & $3.98(2.12)$ & $4.19(2.17)$ & $2.38(1.70)$ & $1.88(1.54)$ & $1.25(1.32)$ & 9.68 \\
\hline 4. & $17^{\text {th }}$ Mar & $4.88(2.32)$ & $4.50(2.24)$ & $2.13(1.62)$ & $2.38(1.70)$ & $1.68(1.47)$ & 10.72 \\
\hline 5. & $27^{\text {th }}$ Mar & $5.81(2.51)$ & $5.55(2.46)$ & $3.13(1.90)$ & $2.88(1.84)$ & $2.13(1.62)$ & 12.12 \\
\hline \multicolumn{2}{|c|}{ SEm \pm} & 0.05 & 0.08 & 0.09 & 0.11 & 0.1 & \\
\hline \multicolumn{2}{|c|}{$\mathrm{CD}(p=0.05)$} & 0.17 & 0.23 & 0.27 & 0.34 & 0.31 & \\
\hline
\end{tabular}

${ }^{*}$ Mean of four replications, ${ }^{* *}$ Figures in parentheses are $\sqrt{ } x+0.5$ transformed values

\begin{tabular}{|c|c|c|c|c|c|c|c|c|c|c|c|c|c|c|}
\hline \multirow{2}{*}{$\begin{array}{l}\text { SI. } \\
\text { No. }\end{array}$} & \multirow{2}{*}{$\begin{array}{l}\text { Treat- } \\
\text { ments }\end{array}$} & \multicolumn{12}{|c|}{ Population*/3leaves in different standard weeks } & \multirow[b]{2}{*}{26} \\
\hline & & 14 & 15 & 16 & 17 & 18 & 19 & 20 & 21 & 22 & 23 & 24 & 25 & \\
\hline 1. & $15^{\text {th }}$ Feb & $\begin{array}{c}9.05 \\
(3.09)^{* *}\end{array}$ & $\begin{array}{l}10.35 \\
(3.29)\end{array}$ & $\begin{array}{l}11.25 \\
(3.43)\end{array}$ & $\begin{array}{l}12.25 \\
(3.57)\end{array}$ & $\begin{array}{l}17.13 \\
(4.20)\end{array}$ & $\begin{array}{l}21.63 \\
(4.70)\end{array}$ & $\begin{array}{l}15.18 \\
(3.96)\end{array}$ & $\begin{array}{l}15.75 \\
(4.03)\end{array}$ & $\begin{array}{l}14.13 \\
(3.82)\end{array}$ & $\begin{array}{l}12.13 \\
(3.55)\end{array}$ & $\begin{array}{c}7.75 \\
(2.87)\end{array}$ & $\begin{array}{c}6.25 \\
(2.60)\end{array}$ & $\begin{array}{c}3.19 \\
(1.92)\end{array}$ \\
\hline 2. & $25^{\text {th }}$ Feb & $\begin{array}{l}10.25 \\
(3.28)\end{array}$ & $\begin{array}{l}12.06 \\
(3.54)\end{array}$ & $\begin{array}{l}12.80 \\
(3.65)\end{array}$ & $\begin{array}{l}13.38 \\
(3.72)\end{array}$ & $\begin{array}{l}18.25 \\
(4.33)\end{array}$ & $\begin{array}{l}23.38 \\
(4.89)\end{array}$ & $\begin{array}{l}17.63 \\
(4.26)\end{array}$ & $\begin{array}{l}16.25 \\
(4.09)\end{array}$ & $\begin{array}{l}15.50 \\
(4.00)\end{array}$ & $\begin{array}{l}13.38 \\
(3.72)\end{array}$ & $\begin{array}{c}9.13 \\
(3.10)\end{array}$ & $\begin{array}{c}7.13 \\
(2.76)\end{array}$ & $\begin{array}{c}4.63 \\
(2.26)\end{array}$ \\
\hline 3. & $7^{\text {th }}$ Mar & $\begin{array}{l}11.19 \\
(3.42)\end{array}$ & $\begin{array}{l}13.36 \\
(3.72)\end{array}$ & $\begin{array}{l}14.25 \\
(3.84)\end{array}$ & $\begin{array}{l}14.63 \\
(3.89)\end{array}$ & $\begin{array}{l}20.50 \\
(4.58)\end{array}$ & $\begin{array}{l}26.50 \\
(5.20)\end{array}$ & $\begin{array}{l}19.13 \\
(4.43)\end{array}$ & $\begin{array}{l}17.25 \\
(4.21)\end{array}$ & $\begin{array}{l}17.55 \\
(4.25)\end{array}$ & $\begin{array}{l}15.23 \\
(3.97)\end{array}$ & $\begin{array}{l}10.75 \\
(3.35)\end{array}$ & $\begin{array}{c}7.94 \\
(2.90)\end{array}$ & $\begin{array}{c}5.38 \\
(2.42)\end{array}$ \\
\hline 4. & $17^{\text {th }}$ Mar & $\begin{array}{l}12.50 \\
(3.61)\end{array}$ & $\begin{array}{l}14.13 \\
(3.82)\end{array}$ & $\begin{array}{l}14.68 \\
(3.90)\end{array}$ & $\begin{array}{l}16.23 \\
(4.09)\end{array}$ & $\begin{array}{l}21.25 \\
(4.66)\end{array}$ & $\begin{array}{l}27.18 \\
(5.26)\end{array}$ & $\begin{array}{l}20.50 \\
(4.58)\end{array}$ & $\begin{array}{l}19.50 \\
(4.47)\end{array}$ & $\begin{array}{l}19.25 \\
(4.44)\end{array}$ & $\begin{array}{l}17.88 \\
(4.29)\end{array}$ & $\begin{array}{l}12.50 \\
(3.61)\end{array}$ & $\begin{array}{c}8.50 \\
(3.00)\end{array}$ & $\begin{array}{c}7.30 \\
(2.79)\end{array}$ \\
\hline 5. & $27^{\text {th }}$ Mar & $\begin{array}{l}12.88 \\
(3.66)\end{array}$ & $\begin{array}{l}15.25 \\
(3.97)\end{array}$ & $\begin{array}{l}15.85 \\
(4.04)\end{array}$ & $\begin{array}{l}17.30 \\
(4.22)\end{array}$ & $\begin{array}{l}23.18 \\
(4.87)\end{array}$ & $\begin{array}{l}29.50 \\
(5.48)\end{array}$ & $\begin{array}{l}23.10 \\
(4.86)\end{array}$ & $\begin{array}{l}21.50 \\
(4.69)\end{array}$ & $\begin{array}{l}21.13 \\
(4.65)\end{array}$ & $\begin{array}{l}19.68 \\
(4.49)\end{array}$ & $\begin{array}{l}13.88 \\
(3.79)\end{array}$ & $\begin{array}{l}11.25 \\
(3.43)\end{array}$ & $\begin{array}{c}8.68 \\
(3.03)\end{array}$ \\
\hline \multicolumn{2}{|c|}{ SEm \pm} & 0.04 & 0.07 & 0.04 & 0.05 & 0.04 & 0.08 & 0.06 & 0.08 & 0.08 & 0.08 & 0.07 & 0.07 & 0.09 \\
\hline \multicolumn{2}{|c|}{$C D(p=0.05)$} & 0.12 & 0.20 & 0.12 & 0.14 & 0.13 & 0.25 & 0.18 & 0.24 & 0.24 & 0.26 & 0.22 & 0.23 & 0.28 \\
\hline \multicolumn{15}{|c|}{ Table 2: Continue... } \\
\hline \multirow{2}{*}{$\begin{array}{l}\text { SI. } \\
\text { No. }\end{array}$} & Treat- & \multicolumn{12}{|c|}{ Population*/3 leaves in different standard weeks } & Over \\
\hline & & 27 & 28 & & 29 & 30 & & 31 & 32 & 33 & 34 & 35 & 36 & mean \\
\hline 1. & $15^{\text {th }}$ Feb & $\begin{array}{c}5.38 \\
(2.42)\end{array}$ & $\begin{array}{c}7.18 \\
(2.77)\end{array}$ & & $\begin{array}{c}3.75 \\
(2.06)\end{array}$ & $\begin{array}{c}1.80 \\
(1.52)\end{array}$ & & $\begin{array}{c}1.13 \\
(1.27)\end{array}$ & $\begin{array}{l}1.98 \\
(1.57)\end{array}$ & $\begin{array}{l}1.23 \\
(1.31)\end{array}$ & $\begin{array}{l}1.13 \\
(1.27)\end{array}$ & $\begin{array}{l}1.28 \\
(1.33)\end{array}$ & $\begin{array}{l}1.15 \\
(1.28)\end{array}$ & 7.91 \\
\hline 2. & $25^{\text {th }}$ Feb & $\begin{array}{c}6.38 \\
(2.62)\end{array}$ & $\begin{array}{c}8.25 \\
(2.96)\end{array}$ & & $\begin{array}{c}4.38 \\
(2.21)\end{array}$ & $\begin{array}{c}2.10 \\
(1.61)\end{array}$ & & $\begin{array}{c}1.25 \\
(1.32)\end{array}$ & $\begin{array}{l}2.38 \\
(1.70)\end{array}$ & $\begin{array}{l}2.38 \\
(1.70)\end{array}$ & $\begin{array}{l}1.38 \\
(1.37)\end{array}$ & $\begin{array}{l}1.85 \\
(1.53)\end{array}$ & $\begin{array}{l}1.25 \\
(1.32)\end{array}$ & 8.93 \\
\hline 3. & $7^{\text {th }}$ Mar & $\begin{array}{c}7.44 \\
(2.82)\end{array}$ & $\begin{array}{c}9.75 \\
(3.20)\end{array}$ & & $\begin{array}{c}5.81 \\
(2.51)\end{array}$ & $\begin{array}{c}4.41 \\
(2.22)\end{array}$ & & $\begin{array}{c}2.25 \\
(1.66)\end{array}$ & $\begin{array}{l}3.68 \\
(2.04)\end{array}$ & $\begin{array}{l}3.06 \\
(1.89)\end{array}$ & $\begin{array}{l}2.13 \\
(1.62)\end{array}$ & $\begin{array}{l}2.50 \\
(1.73)\end{array}$ & $\begin{array}{l}2.74 \\
(1.80)\end{array}$ & 10.32 \\
\hline 4. & $17^{\text {th }} \mathrm{Mar}$ & $\begin{array}{c}8.50 \\
(3.00)\end{array}$ & $\begin{array}{l}12.05 \\
(3.54)\end{array}$ & & $\begin{array}{c}7.50 \\
(2.83)\end{array}$ & $\begin{array}{c}5.25 \\
(2.40)\end{array}$ & & $\begin{array}{c}3.25 \\
(1.94)\end{array}$ & $\begin{array}{l}5.05 \\
(2.36)\end{array}$ & $\begin{array}{l}4.25 \\
(2.18)\end{array}$ & $\begin{array}{l}3.63 \\
(2.03)\end{array}$ & $\begin{array}{l}3.38 \\
(1.97)\end{array}$ & $\begin{array}{l}2.98 \\
(1.86)\end{array}$ & 11.62 \\
\hline 5. & $27^{\text {th }} \mathrm{Mar}$ & $\begin{array}{l}10.63 \\
(3.34)\end{array}$ & $\begin{array}{l}13.93 \\
(3.80)\end{array}$ & & $\begin{array}{c}9.25 \\
(3.12)\end{array}$ & $\begin{array}{c}6.23 \\
(2.59)\end{array}$ & & $\begin{array}{c}3.93 \\
(2.10)\end{array}$ & $\begin{array}{l}5.93 \\
(2.53)\end{array}$ & $\begin{array}{l}5.75 \\
(2.50)\end{array}$ & $\begin{array}{l}4.38 \\
(2.21)\end{array}$ & $\begin{array}{l}4.18 \\
(2.16)\end{array}$ & $\begin{array}{l}3.75 \\
(2.06)\end{array}$ & 13.09 \\
\hline SEm: & & 0.09 & 0.08 & & 0.09 & 0.06 & & 0.05 & 0.06 & 0.06 & 0.06 & 0.05 & 0.06 & \\
\hline CD $(1$ & $=0.05)$ & 0.27 & 0.25 & & 0.27 & 0.17 & & 0.16 & 0.19 & 0.18 & 0.18 & 0.16 & 0.17 & \\
\hline
\end{tabular}

${ }^{*}$ Mean of four replications, ${ }^{* *}$ Figures in parentheses are $\mathrm{V} x+0.5$ transformed values 
in all dates of transplanting $\left(15^{\text {th }}\right.$ Feb, $25^{\text {th }}$ Feb, $7^{\text {th }}$ March, $11^{\text {th }}$ March, $27^{\text {th }}$ March), thereafter, increased gradually and reached to its peak in the month of July and then declined gradually. At the peak the maximum population (7.03/three leaves) of jassid were recorded on the crop transplanting on $27^{\text {th }}$ March and minimum (3.14/three leaves) on $15^{\text {th }}$ Feb was observed then followed by 3.68, 4.40 and 5.73/three leaves in $25^{\text {th }} \mathrm{Feb}, 7^{\text {th }}$ March and $17^{\text {th }}$ March transplanting crop respectively (Table 3 ). Like were the jassid population was also recorded minimum on the crop transplanted early $\left(15^{\text {th }}\right.$ Feb.) and the maximum on the late transplanted crop $\left(27^{\text {th }}\right.$ March) during both the years. The present results corroborate with that of Nagargoje et al. (2002) who reported that jassid incidence was increased as sowing dates delayed.

\subsection{Mite}

The population of mite remained low throughout the crop season in all dates of transplanting. The overall mean of mite population recorded during the season in all dates of transplanting ranged from 1.72 to 5.74 mite per three leaves. The minimum population was found on the crop transplanted on $15^{\text {th }}$ Feb. followed by $25^{\text {th }}$ Feb. transplanted crop. The maximum number of mites observed from the crop transplanted on $27^{\text {th }}$ March followed by $17^{\text {th }}$ march transplanted crop (Table 4). The present result are inconformity with those of Murthy (1984) and Lingeri et al. (1998 a\&b) who reported that mite population was recorded less in early sown crop as compared to late sown chilli crops.

3.5. Aphid

\begin{tabular}{|c|c|c|c|c|c|c|c|c|c|c|c|c|c|c|}
\hline \multirow{2}{*}{$\begin{array}{l}\text { Sl. } \\
\text { No. }\end{array}$} & \multirow{2}{*}{$\begin{array}{l}\text { Treat- } \\
\text { ments }\end{array}$} & \multicolumn{12}{|c|}{ Population*/3leaves in different standard weeks } & \multirow[b]{2}{*}{29} \\
\hline & & 17 & 18 & 19 & 20 & 21 & 22 & 23 & 24 & 25 & 26 & 27 & 28 & \\
\hline 1. & $15^{\text {th }} \mathrm{Feb}$ & $\begin{array}{c}0.31 \\
(0.90)^{* *}\end{array}$ & $\begin{array}{c}1.93 \\
(1.56)\end{array}$ & $\begin{array}{c}1.81 \\
(1.52)\end{array}$ & $\begin{array}{c}1.55 \\
(1.43)\end{array}$ & $\begin{array}{c}1.19 \\
(1.30)\end{array}$ & $\begin{array}{c}1.61 \\
(1.45)\end{array}$ & $\begin{array}{c}2.75 \\
(1.80)\end{array}$ & $\begin{array}{c}1.25 \\
(1.32)\end{array}$ & $\begin{array}{c}2.63 \\
(1.77)\end{array}$ & $\begin{array}{c}4.88 \\
(2.32)\end{array}$ & $\begin{array}{c}4.05 \\
(2.13)\end{array}$ & $\begin{array}{c}6.75 \\
(2.69)\end{array}$ & $\begin{array}{r}9.50 \\
(3.16)\end{array}$ \\
\hline 2. & $25^{\text {th }}$ Feb & $\begin{array}{c}0.56 \\
(1.03)\end{array}$ & $\begin{array}{c}2.19 \\
(1.64)\end{array}$ & $\begin{array}{c}2.25 \\
(1.66)\end{array}$ & $\begin{array}{c}1.68 \\
(1.47)\end{array}$ & $\begin{array}{c}1.94 \\
(1.56)\end{array}$ & $\begin{array}{c}2.13 \\
(1.62)\end{array}$ & $\begin{array}{c}3.50 \\
(2.00)\end{array}$ & $\begin{array}{c}2.13 \\
(1.62)\end{array}$ & $\begin{array}{c}3.25 \\
(1.94)\end{array}$ & $\begin{array}{c}5.75 \\
(2.50)\end{array}$ & $\begin{array}{c}4.25 \\
(2.18)\end{array}$ & $\begin{array}{c}7.38 \\
(2.81)\end{array}$ & $\begin{array}{l}10.50 \\
(3.32)\end{array}$ \\
\hline 3. & $7^{\text {th }}$ Mar & $\begin{array}{c}0.69 \\
(1.09)\end{array}$ & $\begin{array}{c}2.63 \\
(1.77)\end{array}$ & $\begin{array}{c}2.75 \\
(1.80)\end{array}$ & $\begin{array}{c}2.63 \\
(1.77)\end{array}$ & $\begin{array}{c}2.05 \\
(1.60)\end{array}$ & $\begin{array}{c}2.75 \\
(1.80)\end{array}$ & $\begin{array}{c}3.75 \\
(2.06)\end{array}$ & $\begin{array}{c}2.56 \\
(1.75)\end{array}$ & $\begin{array}{c}3.06 \\
(1.89)\end{array}$ & $\begin{array}{c}5.93 \\
(2.53)\end{array}$ & $\begin{array}{c}4.63 \\
(2.26)\end{array}$ & $\begin{array}{c}8.50 \\
(3.00)\end{array}$ & $\begin{array}{l}13.68 \\
(3.76)\end{array}$ \\
\hline 4. & $17^{\text {th }}$ Mar & $\begin{array}{c}1.11 \\
(1.27)\end{array}$ & $\begin{array}{c}3.44 \\
(1.98)\end{array}$ & $\begin{array}{c}3.30 \\
(1.95)\end{array}$ & $\begin{array}{c}2.38 \\
(1.70)\end{array}$ & $\begin{array}{c}4.65 \\
(2.27)\end{array}$ & $\begin{array}{c}3.25 \\
(1.94)\end{array}$ & $\begin{array}{c}4.75 \\
(2.29)\end{array}$ & $\begin{array}{c}3.25 \\
(1.94)\end{array}$ & $\begin{array}{c}4.88 \\
(2.32)\end{array}$ & $\begin{array}{c}6.25 \\
(2.60)\end{array}$ & $\begin{array}{c}5.63 \\
(2.47)\end{array}$ & $\begin{array}{l}10.75 \\
(3.35)\end{array}$ & $\begin{array}{l}15.73 \\
(4.03)\end{array}$ \\
\hline 5. & $27^{\text {th }}$ Mar & $\begin{array}{c}1.38 \\
(1.37)\end{array}$ & $\begin{array}{c}4.24 \\
(2.18)\end{array}$ & $\begin{array}{c}4.06 \\
(2.14)\end{array}$ & $\begin{array}{c}2.50 \\
(1.73)\end{array}$ & $\begin{array}{c}6.25 \\
(2.60)\end{array}$ & $\begin{array}{c}5.13 \\
(2.37)\end{array}$ & $\begin{array}{c}6.05 \\
(2.56)\end{array}$ & $\begin{array}{c}4.13 \\
(2.15)\end{array}$ & $\begin{array}{c}6.38 \\
(2.62)\end{array}$ & $\begin{array}{c}7.69 \\
(2.86)\end{array}$ & $\begin{array}{c}6.50 \\
(2.65)\end{array}$ & $\begin{array}{l}13.00 \\
(3.67)\end{array}$ & $\begin{array}{l}18.63 \\
(4.37)\end{array}$ \\
\hline \multicolumn{2}{|c|}{ SEm \pm} & 0.08 & 0.05 & 0.06 & 0.06 & 0.07 & 0.06 & 0.08 & 0.05 & 0.09 & 0.06 & 0.09 & 0.07 & 0.10 \\
\hline \multicolumn{2}{|c|}{$\operatorname{CD}(p=0.05)$} & 0.25 & 0.16 & 0.19 & 0.17 & 0.23 & 0.18 & 0.25 & 0.15 & 0.27 & 0.20 & 0.27 & 0.20 & 0.30 \\
\hline \multicolumn{15}{|c|}{ Table 3: Continue... } \\
\hline \multirow{2}{*}{$\begin{array}{l}\text { SI. } \\
\text { No. }\end{array}$} & \multirow{2}{*}{\multicolumn{2}{|c|}{ Treatments }} & \multicolumn{10}{|c|}{ Population*/3 leaves in different standard weeks } & \multirow{2}{*}{\multicolumn{2}{|c|}{$\begin{array}{c}\text { Over all } \\
\text { mean }\end{array}$}} \\
\hline & & & 30 & & 31 & & 32 & & 33 & 34 & & 35 & & \\
\hline 1. & $15^{\text {th }} \mathrm{Feb}$ & & $\begin{array}{c}8.18 \\
(2.95)\end{array}$ & & $\begin{array}{c}6.25 \\
(2.60)\end{array}$ & & $\begin{array}{c}2.80 \\
(1.82)\end{array}$ & & $\begin{array}{c}1.69 \\
(1.48)\end{array}$ & $\begin{array}{r}1.05 \\
(1.24\end{array}$ & & $\begin{array}{l}0.43 \\
(0.96)\end{array}$ & \multicolumn{2}{|r|}{3.14} \\
\hline 2. & $25^{\text {th }}$ Feb & & $\begin{array}{c}9.10 \\
(3.10)\end{array}$ & & $\begin{array}{c}7.38 \\
(2.81)\end{array}$ & & $\begin{array}{c}3.06 \\
(1.89)\end{array}$ & & $\begin{array}{c}2.05 \\
(1.60)\end{array}$ & $\begin{array}{r}1.18 \\
(1.29\end{array}$ & & $\begin{array}{l}0.63 \\
(1.06)\end{array}$ & \multicolumn{2}{|r|}{3.68} \\
\hline 3. & $7^{\text {th }}$ Mar & & $\begin{array}{l}10.25 \\
(3.28)\end{array}$ & & $\begin{array}{c}8.88 \\
(3.06)\end{array}$ & & $\begin{array}{c}4.05 \\
(2.13)\end{array}$ & & $\begin{array}{c}3.60 \\
(2.02)\end{array}$ & $\begin{array}{r}2.50 \\
(1.73\end{array}$ & & $\begin{array}{l}1.80 \\
(1.52)\end{array}$ & \multicolumn{2}{|r|}{4.40} \\
\hline 4. & $17^{\text {th }}$ Mar & & $\begin{array}{l}13.93 \\
(3.80)\end{array}$ & & $\begin{array}{l}10.35 \\
(3.29)\end{array}$ & & $\begin{array}{c}5.98 \\
(2.54)\end{array}$ & & $\begin{array}{c}4.80 \\
(2.30)\end{array}$ & $\begin{array}{r}4.25 \\
(2.18\end{array}$ & & $\begin{array}{l}3.25 \\
(1.94)\end{array}$ & \multicolumn{2}{|r|}{5.73} \\
\hline 5. & $27^{\text {th }}$ Mar & & $\begin{array}{l}15.63 \\
(4.02)\end{array}$ & & $\begin{array}{l}12.05 \\
(3.54)\end{array}$ & & $\begin{array}{c}8.3 \\
(2.97)\end{array}$ & & $\begin{array}{c}5.63 \\
(2.47)\end{array}$ & $\begin{array}{r}5.50 \\
(2.45\end{array}$ & & $\begin{array}{l}5.05 \\
(2.36)\end{array}$ & \multicolumn{2}{|r|}{7.06} \\
\hline \multicolumn{2}{|c|}{ SEm \pm} & & 0.07 & & 0.09 & & 0.07 & & 0.06 & 0.08 & & 0.06 & & \\
\hline $\mathrm{CD}(p$ & $0.05)$ & & 0.22 & & 0.27 & & 0.22 & & 0.20 & 0.24 & & 0.18 & & \\
\hline
\end{tabular}

${ }^{*}$ Mean of four replications, ${ }^{* *}$ Figures in parentheses are $\mathrm{V} x+0.5$ transformed values 
The aphid incidence was started on chilli crop just after transplanting in all dates of transplanting. The data indicated that it remained on chilli crop only up to last week of April thereafter, it could not be observed on chilli crop transplanted on different dates throughout the season. At the peak, means first week of April, the minimum number (3.71/three leaves)

\begin{tabular}{|c|c|c|c|c|c|c|c|c|c|c|c|c|c|c|}
\hline \multirow{2}{*}{$\begin{array}{l}\text { SI. } \\
\text { No. }\end{array}$} & \multirow{2}{*}{$\begin{array}{l}\text { Treat- } \\
\text { ments }\end{array}$} & \multicolumn{12}{|c|}{ Population*/3leaves in different standard weeks } & \multirow[b]{2}{*}{25} \\
\hline & & 13 & 14 & 15 & 16 & 17 & 18 & 19 & 20 & 21 & 22 & 23 & 24 & \\
\hline 1. & $15^{\text {th }} \mathrm{Feb}$ & $\begin{array}{c}1.60 \\
(1.45)^{* *}\end{array}$ & $\begin{array}{c}2.13 \\
(1.62)\end{array}$ & $\begin{array}{c}2.44 \\
(1.71)\end{array}$ & $\begin{array}{c}2.50 \\
(1.73)\end{array}$ & $\begin{array}{c}2.06 \\
(1.60)\end{array}$ & $\begin{array}{c}1.63 \\
(1.46)\end{array}$ & $\begin{array}{c}1.56 \\
(1.44)\end{array}$ & $\begin{array}{c}1.88 \\
(1.54)\end{array}$ & $\begin{array}{c}2.98 \\
(1.86)\end{array}$ & $\begin{array}{c}1.06 \\
(1.25)\end{array}$ & $\begin{array}{c}0.55 \\
(1.02)\end{array}$ & $\begin{array}{c}0.63 \\
(1.06)\end{array}$ & $\begin{array}{c}1.13 \\
(1.27)\end{array}$ \\
\hline 2. & $25^{\text {th }} \mathrm{Feb}$ & $\begin{array}{c}2.31 \\
(1.68)\end{array}$ & $\begin{array}{c}3.30 \\
(1.95)\end{array}$ & $\begin{array}{c}3.24 \\
(1.93)\end{array}$ & $\begin{array}{c}3.24 \\
(1.93)\end{array}$ & $\begin{array}{c}2.38 \\
(1.70)\end{array}$ & $\begin{array}{c}2.05 \\
(1.60)\end{array}$ & $\begin{array}{c}2.25 \\
(1.66)\end{array}$ & $\begin{array}{c}2.38 \\
(1.70)\end{array}$ & $\begin{array}{c}3.50 \\
(2.00)\end{array}$ & $\begin{array}{c}2.00 \\
(1.58)\end{array}$ & $\begin{array}{c}0.80 \\
(1.14)\end{array}$ & $\begin{array}{c}0.93 \\
(1.19)\end{array}$ & $\begin{array}{c}2.50 \\
(1.73)\end{array}$ \\
\hline 3. & $7^{\text {th }}$ Mar & $\begin{array}{c}2.88 \\
(1.84)\end{array}$ & $\begin{array}{c}4.19 \\
(2.17)\end{array}$ & $\begin{array}{c}4.13 \\
(2.15)\end{array}$ & $\begin{array}{c}3.93 \\
(2.10)\end{array}$ & $\begin{array}{c}3.18 \\
(1.92)\end{array}$ & $\begin{array}{c}2.60 \\
(1.76)\end{array}$ & $\begin{array}{c}3.68 \\
(2.04)\end{array}$ & $\begin{array}{c}3.18 \\
(1.92)\end{array}$ & $\begin{array}{c}4.48 \\
(2.23)\end{array}$ & $\begin{array}{c}3.18 \\
(1.92)\end{array}$ & $\begin{array}{c}1.49 \\
(1.41)\end{array}$ & $\begin{array}{c}1.60 \\
(1.45)\end{array}$ & $\begin{array}{c}3.23 \\
(1.93)\end{array}$ \\
\hline 4. & $17^{\text {th }}$ Mar & $\begin{array}{c}3.43 \\
(1.98)\end{array}$ & $\begin{array}{c}5.75 \\
(2.50)\end{array}$ & $\begin{array}{c}5.68 \\
(2.48)\end{array}$ & $\begin{array}{c}5.18 \\
(2.38)\end{array}$ & $\begin{array}{c}4.18 \\
(2.16)\end{array}$ & $\begin{array}{c}3.63 \\
(2.03)\end{array}$ & $\begin{array}{c}4.68 \\
(2.27)\end{array}$ & $\begin{array}{c}4.28 \\
(2.19)\end{array}$ & $\begin{array}{c}6.55 \\
(2.66)\end{array}$ & $\begin{array}{c}4.18 \\
(2.16)\end{array}$ & $\begin{array}{c}2.23 \\
(1.65)\end{array}$ & $\begin{array}{c}3.30 \\
(1.95)\end{array}$ & $\begin{array}{c}3.50 \\
(2.00)\end{array}$ \\
\hline 5. & $27^{\text {th }}$ Mar & $\begin{array}{c}4.88 \\
(2.32)\end{array}$ & $\begin{array}{c}6.75 \\
(2.69)\end{array}$ & $\begin{array}{c}7.13 \\
(2.76)\end{array}$ & $\begin{array}{c}7.75 \\
(2.87)\end{array}$ & $\begin{array}{c}5.10 \\
(2.37)\end{array}$ & $\begin{array}{c}5.25 \\
(2.40)\end{array}$ & $\begin{array}{c}6.38 \\
(2.62)\end{array}$ & $\begin{array}{c}6.13 \\
(2.57)\end{array}$ & $\begin{array}{c}7.55 \\
(2.84)\end{array}$ & $\begin{array}{c}5.23 \\
(2.39)\end{array}$ & $\begin{array}{c}3.50 \\
(2.00)\end{array}$ & $\begin{array}{c}4.13 \\
(2.15)\end{array}$ & $\begin{array}{c}4.63 \\
(2.26)\end{array}$ \\
\hline \multicolumn{2}{|c|}{ SEm \pm} & 0.07 & 0.09 & 0.07 & 0.08 & 0.04 & 0.04 & 0.08 & 0.06 & 0.06 & 0.06 & 0.04 & 0.05 & 0.05 \\
\hline \multicolumn{2}{|c|}{$\mathrm{CD}(p=0.05)$} & 0.22 & 0.27 & 0.21 & 0.25 & 0.12 & 0.13 & 0.24 & 0.19 & 0.18 & 0.19 & 0.13 & 0.15 & 0.16 \\
\hline
\end{tabular}

Table 4: Continue...

SI. Treat- Population*/3leaves in dif- Over all No. ments ferent standard weeks Mean

\begin{tabular}{lcccc} 
& & 26 & 27 & \\
\cline { 3 - 4 } 1. & $15^{\text {th }}$ Feb & $2.50(1.73)$ & $1.13(1.27)$ & 1.72 \\
2. & $25^{\text {th }}$ Feb & $3.38(1.97)$ & $1.50(1.41)$ & 2.40 \\
3. & $7^{\text {th }}$ Mar & $4.18(2.16)$ & $2.05(1.60)$ & 3.20 \\
4. & $17^{\text {th }}$ Mar & $6.30(2.61)$ & $3.75(2.06)$ & 4.44 \\
5. & $27^{\text {th }}$ Mar & $6.75(2.69)$ & $4.93(2.33)$ & 5.74 \\
SEm \pm & 0.09 & 0.09 & \\
\multicolumn{2}{l}{ CD $(p=0.05)$} & 0.27 & 0.29 & \\
\hline
\end{tabular}

"Mean of four replications, ${ }^{* *}$ Figures in parentheses are $v \mathrm{x}+0.5$ transformed values of aphid recorded on the crop transplanted on $15^{\text {th }}$ Feb. which was found at par to the crop transplanted on $25^{\text {th }} \mathrm{Feb}$. The highest population (9.11/three leaves) was observed on the crop transplanted on $27^{\text {th }}$ March followed by the crop transplanted in $17^{\text {th }}$ March (Table 5). However these were at per to each other. The data obtained in the present findings indicated that there was a definite pattern of aphid incidence in relation to different dates of transplanting of chilli. It was apparent that there was corporately best incidence of aphids on chilli crop in $15^{\text {th }}$ Feb. (early sowing). The later transplanting crops were progressively more infested and these results are in close conformity with the findings of Nagargoje et al. (2002) who has reported the maximum population of aphids on late transplanting crop of chilli.

Table 5: Effect of dates of transplanting on the incidence of aphid during summer 2010

\begin{tabular}{llccccc}
\hline Sl. No. & Treatments & \multicolumn{3}{c}{ Population*/3leaves in different standard weeks } & Mean \\
\cline { 3 - 5 } & & 13 & 14 & 15 & 16 & \\
\hline 1. & $15^{\text {th }} \mathrm{Feb}$ & $8.13(2.94)^{* *}$ & $5.55(2.46)$ & $0.73(1.11)$ & $0.43(0.96)$ & 3.71 \\
2. & $25^{\text {th }} \mathrm{Feb}$ & $9.25(3.12)$ & $6.18(2.58)$ & $1.38(1.37)$ & $0.60(1.05)$ & 4.35 \\
3. & $7^{\text {th }} \mathrm{Mar}$ & $10.75(3.35)$ & $9.50(3.16)$ & $2.68(1.78)$ & $2.19(1.64)$ & 6.28 \\
4. & $17^{\text {th }}$ Mar & $13.40(3.73)$ & $10.38(3.30)$ & $4.38(2.21)$ & $3.18(1.92)$ & 7.83 \\
5. & $27^{\text {th }}$ Mar & $14.55(3.88)$ & $11.93(3.52)$ & $5.63(2.47)$ & $4.35(2.20)$ & 9.11 \\
SEm \pm & & 0.10 & 0.07 & 0.07 & 0.06 & \\
CD $(p=0.05)$ & & 0.31 & 0.21 & 0.23 & 0.19 & \\
\hline
\end{tabular}

"Mean of four replications, ${ }^{* *}$ Figures in parentheses are $\sqrt{ } x+0.5$ transformed values 


\section{Conclusion}

The experiment on five dates of transplanting $15^{\text {th }}$ Feb., $25^{\text {th }}$ Feb., $7^{\text {th }}$ March, $17^{\text {th }}$ March and $27^{\text {th }}$ March were evaluated on occurrence of major sucking pests of chilli i.e. thrips (Scirtothrips dorsalis Hood), whitefly (Bemisia tabaci Genn.), aphid (Aphis gossypii Glover), jassid (Amrasca biguttula biguttula Ishida) and mite (Polyphagotarsonemus latus Banks). The experiment on five dates of transplanting revealed that early transplanting $15^{\text {th }}$ Feb. had the minimum and $27^{\text {th }}$ March had maximum infestation of sucking pests viz., thrips, whitefly, jassid, mite and aphid, and highest green chilli yield on compared to the late transplanted crop $27^{\text {th }}$ march.

\section{References}

Anonymous, 2015. Agricultural Statistics at a Glance Government of India Ministry of Agriculture Department of Agriculture and Cooperation Directorate of Economics and Statistics.

Anonymous, 2015. Rajasthan Agricultural Statistics at a glance 2016-17, 108.

Bishnoi, O.P., Singh, M., Rao, V.U.M., Niwas, R., Sharma, P.D., 1996. Population dynamics of cotton pests in relation to weather parameter. Indian Journal of Entomology 58(2), 103-107.

Borah, R.K., Langthasa, S., 1995. Incidence of thrips scirtothrips dorsalis Hood in relation of data of transplanting on chilli in hill zone of Assam. PKV Research Journal 19(1), 92.

Dhawan, A.K., Simwat, G.S., Dhaliwal, G.S., 1998. Population dynamics of whitefly. Bemisia tabaci (Gennadius) on cotton: an eco-behavioural approach. Ecological agriculture and sustainable development Vol.1 Proc. int. Conf. Eco. Agri. Towards Sus. Dev. Chandigarh India. 435-448.

Giri, D.G., Daware, D.G., Dahiphale, V.V., 1993. Population level of whitefly (Bemisia tabaci) as influenced by different agronomic regime J. Cotton Res. \& Dev., 7(1), 168-169.

Hosmani, M.M., 1982. Cultural practices for chilli, In: Chilli edited by Hosmani, M.M., Rajashri Printing press, Tadakocbni, Dharwad, 100-128.

Lingeri, M.S., Awaknavar, J.S., Lingappa, S., Kulkarni, K.A., 1998. Seasonal occurrence of chilli mites (Polyphagotarsonemus latus Banks) and thrips (Scirtothrips dorsalis Hood). Karnataka Journal of Agricultural Sciences 11(2), 380-385.

Lingeri, M.S., Awaknavar, K.A., Kulkarni, S., Lingappa, S., Madalagni, B.B., 1998b. Screening of chilli genotypes against polyphegotarsomemens latus (banks) and thrips (Scirtothrips dorsalis Hood.). Karnataka Journal of Agricultural Sciences 11, 39-44.

Murthy, N.S., 1984. Chilli cultivation in Andhra Pradesh India cocoa. Arecanut and spices J., 7, 103-105.

Nagargoje, C., Mehetre, S.S., Patil, S.D., 2002. Effect of different sowing dates and irrigation methods on insect pests of cotton. J. Cotton. Res. Dev., 16 (2), 227-229.

Patel, V.N., Gupta, H.C.L., 1998. Estimation of losses and management of thrips infesting chillies in National Seminar on "Entomology in $21^{\text {st }}$ Century" Biodiversity, Sustainability, Environmental Safety and Human Health pp 99. Rajasthan Agriculture University, Udaipur.

Reddy, D.N.R., Puttaswamy, 1985. Pests infesting chilli (Capsicum annum L.) in the nursery. Mysore journal of agricultural sciences 18(2), 122-125. 